\title{
Grip Force Using an Artificial Limb in a Congenital Amputee
}

\author{
Michael Trujillo, David I. Anderson, Marilyn Mitchell* \\ Department of Kinesiology, San Francisco State University, San Francisco, USA \\ Email: " marilynsfsu@gmail.com
}

Received 18 June 2014; revised 20 July 2014; accepted 2 August 2014

Copyright (C) 2014 by authors and Scientific Research Publishing Inc.

This work is licensed under the Creative Commons Attribution International License (CC BY). http://creativecommons.org/licenses/by/4.0/

(c) (i) Open Access

\section{Abstract}

While much attention is being given in the application of advanced technologies to improve upper extremity prostheses, traditional body-powered prostheses still remain the most popular by people with an amputation. A body-powered prosthesis provides the user with a reasonable solution for limb loss given their simple design, lower maintenance and initial cost. The two major types of body-powered prosthesis use either voluntary opening or voluntary closing control of the terminal device (or prehensor) used for holding and manipulating objects. What differentiates these two types of control is the relationship between the muscular force used to apply tension on the cable attached to the prehensor and the force produced by the prehensor. It has been argued that the voluntary closing prosthesis has more optimal compatibility between the muscle force and grip force of the prehensor. As a result, it may provide an advantage to the user in tasks requiring the control of grip force. To determine the effectiveness of the voluntary closing and voluntary opening prosthesis, we asked a person with a congenital quadruple limb deficiency who is right hand dominant, and that uses voluntary opening prostheses to participate in a study investigating grip force control. The participant was required to match different target grip forces displayed on a computer monitor by manipulating the pressure exerted on a hand dynamometer using either a voluntary closing or voluntary opening prosthesis. The participant only had previous experience with a voluntary opening prosthesis. The results showed that in several measures, the participant performed better with the voluntary closing prosthesis. These results provided support for the muscular force-grip force compatibility hypothesis.

\section{Keywords}

Grip Force, Amputation, Prehensor

\footnotetext{
${ }^{*}$ Corresponding author.
} 


\section{Introduction}

A limb amputation can have detrimental consequences on quality of life. The use of a prosthetic limb can remediate some of those deficits. For upper extremity amputations, where the hand is removed, an upper extremity prosthetic limb can dramatically improve manipulation capabilities for the amputee. The upper extremity prosthetic limb can be fit with a number of prehensors or hands. Some prehensors are chosen for functionality, others are chosen for cosmetic reasons. While prosthetic limb technology has advanced tremendously over recent years, functionality of the prosthetic limb is the ultimate goal [1] and the body-powered prosthesis that utilizes a cabling system, is still the most prevalent. Increases and decreases of the cable tension caused by relative body motions effectively alter the opening and closing of the prehensor. Of the prehensors that are chosen for functionality, the voluntary opening (voluntary opening) terminal device is most commonly prescribed [2]. The voluntary opening device remains closed until tension is produced on an attached cable to open the prehensor. In contrast, the voluntary closing prehensor remains open until cable tension is used to close the prehensor (see Figure 1(a)).

Though the voluntary opening terminal device is the most commonly used, it has been suggested that the voluntary closing is more logically compatible with the functionality of the anatomical, or natural hand [3]-[7]. To increase grip force output with the anatomical hand and the voluntary closing prehensor, a person must increase the force produced by the muscles controlling the hand or those that increase the tension on the cables controlling the voluntary closing prehensor. In other words, for both the anatomical hand and the voluntary closing prehensor, increases in grip force output are controlled by increases in the force produced by the muscles of the

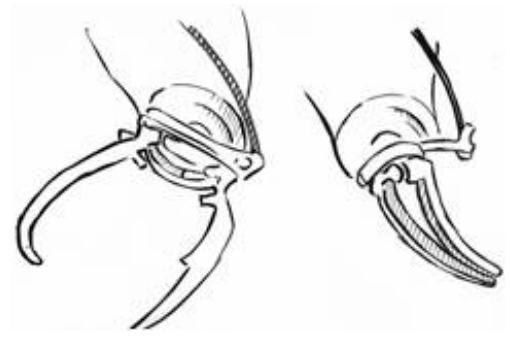

(a)

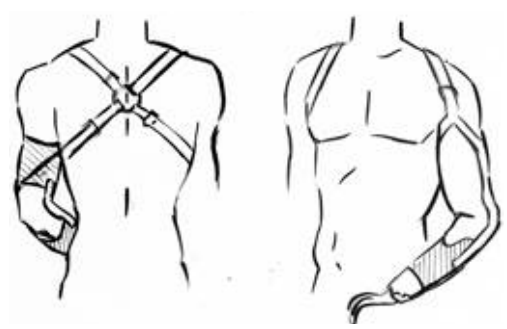

(b)

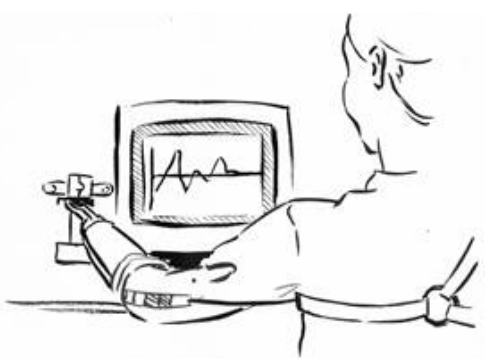

(c)

Figure 1. In (a), shows a schematic of the voluntary closing split hook prehensor (left) and voluntary opening split hook prehensor (right). In (b), shows a schematic of the cabling system and prosthetic limb of an upper extremity amputee. In (c), shows a schematic of a person with an amputation using a prosthesis performing the static grip force experiment. 
anatomical hand, or the muscles controlling cable tension of the voluntary closing device. An analogy that illustrates this type of compatibility is the braking system on a bicycle. Muscular force applied to the hand brakes results in increasing force of the bike pads on the tire. With the voluntary opening, in contrast, increases in voluntary muscular force resulting in increases in cable tension are accompanied by opening the prehensor leading to decreases in prehensor grip force output. Thus, in tasks requiring grip force modulation, the natural compatibility between muscular force and grip force using the voluntary closing or anatomical hand may result in more effective performance compared to the voluntary opening prehensor, particularly to the inexperienced user.

Based on this analysis, a muscular force-grip force compatibility hypothesis has been advanced proposing that in tasks requiring grip force modulation, the voluntary closing prehensor should allow the inexperienced user to perform similar to the anatomical hand, and possibly better compared to the voluntary opening prehensor [7]. However, we had no idea on how an experienced prosthetic user might perform using a voluntary closing prehensor, particularly an individual with considerable experience using a voluntary opening prehensor. The muscular force-grip force compatibility hypothesis would predict good performance with the voluntary closing prehensor because of its superior compatibility, though the specificity of practice principle would predict superior performance with the voluntary opening prehensor because of its familiarity to the user [8]-[10].

The purpose of this case study was to compare accuracy and variability in constant force production in a congenital amputee when using either his native prehensor (voluntary opening) or the voluntary closing prehensor with which he had no previous experience. The person with a congenital limb deficiency has been a voluntary opening user since being introduced to a prosthetic limb as a toddler. We hypothesized that he would be better at modulating grip force with the voluntary closing despite being an experienced voluntary opening user due to the compatibility between muscular force and grip force output in the voluntary closing and anatomical hand. However, we were open to the possibility that being an experienced voluntary opening user, our participant may have learned to overcome the muscular force-grip force incompatibility inherent in his voluntary opening prosthesis.

\section{Methods}

Participant. One 35-year-old male, with a congenital quadruple limb deficiency who is right hand dominant, and uses voluntary opening prostheses with a split-hook prehensor, participated in the experiment. The University's Institutional Review Board approved the study, and the participant provided written informed consent prior to participation.

Procedure. The participant's own below elbow prosthesis on the left, non-dominant, limb was used with his own split hook voluntary opening device (voluntary opening), and with a split hook voluntary closing device that is comparable in size and weight to the voluntary opening device. The prosthesis uses a figure-9 harness fitted around the contralateral limb. The harness attaches to a cable that runs across the back and upper arm ipsilateral to the simulator and is connected to the terminal device (TD). Placing tension on the cable opens the voluntary opening grip and closes the voluntary closing grip (see Figure 1(b)). Correspondingly, as tension on the cable increases, the grip force produced by the voluntary opening grip decreases and the voluntary closing grip increases. Manipulation of the cable tension is carried out through movements of the shoulder contralateral to the prosthesis.

The participant sat in an adjustable height chair at a table where the apparatus was secured. An isometric hand dynamometer was vertically mounted to a table in front of the participant and connected to a Biopac MP30 Student Lab System (Biopac Systems, Inc., Santa Barbara, CA). The hand dynamometer was used to measure constant static grip force at $500 \mathrm{~Hz}$. Force output from the Hand Dynamometer was displayed on a 15 inch CRT monitor that was positioned at eye level one meter from the participant. The software displayed the force ouput generated by squeezing the hand dynamometer, as a horizontal trace, relative to a stationary horizontal target line (see Figure 1(c)). The participant was asked to produce a grip force with his non-dominant left side so that the force output matched the target force of either 0.49 or 10.5 newtons. The initial 5 seconds of each trial was factored out of the analyses to control for variability in reaching the target force. Two positions were assessed, one where the elbow was flexed to $90^{\circ}$ and another where the elbow was extended to $180^{\circ}$. Because the tension on the cable varies as a function of the position of the prehensor relative to the shoulder, we thought that performance may vary in the different arm positions. In the $90^{\circ}$ position, the cable tension biases the voluntary opening prehensor toward opening and the voluntary closing prehensor toward closing. Additionally, force accuracy and variability was assessed with and without vision. For the no-vision conditions, visual feedback of 
force output was taken away after 5 seconds. A total of 48 trials were assessed. Each target force, vision, and position combination was repeated for 3 trials.

Measurements. Three measures were used to assess accuracy and variability. Absolute constant error (CE) is a measure of accuracy and is defined as the average absolute difference between the target force and the produced grip force output over the final 10 seconds of each trial. Variable error (VE) is a measure of variability and is defined as the standard deviation of the difference between the target force and the produced grip force output over the final 10 seconds of each trial. Bias index (BI) is an additional measure of accuracy where the resultant index quantifies the degree to which the participant overshot or undershot the target. BI is calculated as:

$$
\mathrm{BI}=(\text { under }- \text { over }) /(\text { under }+ \text { over })
$$

where over is the amount of time spent over the target and under is the amount of time spent under the target. BI gives values between -1 and 1 , with -1 being a bias toward undershooting the target and values near 1 indicating a bias toward overshooting the target. For each measure, a t-test was used to determine whether there was a significant difference between the 6 trials with the voluntary opening prehensor and the 6 trials with the voluntary closing prehensor for each force $\times$ vision condition.

Figure 2 shows 4 representative traces of force output for the voluntary opening and voluntary closing prehensors, with and without vision at the 10.5 newton target. The corresponding CE, VE, and BI are displayed for each force output trace (Figure 2).

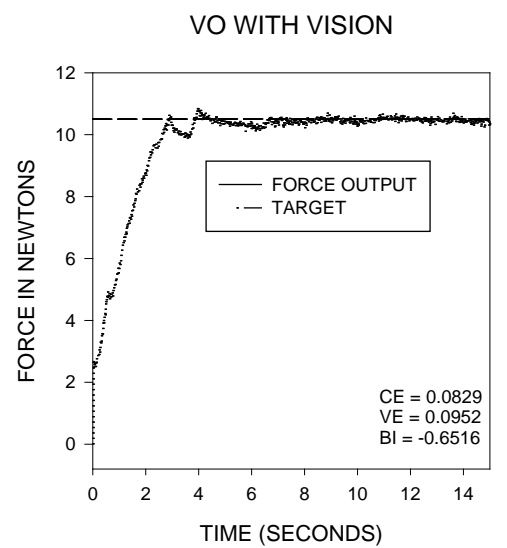

(a)

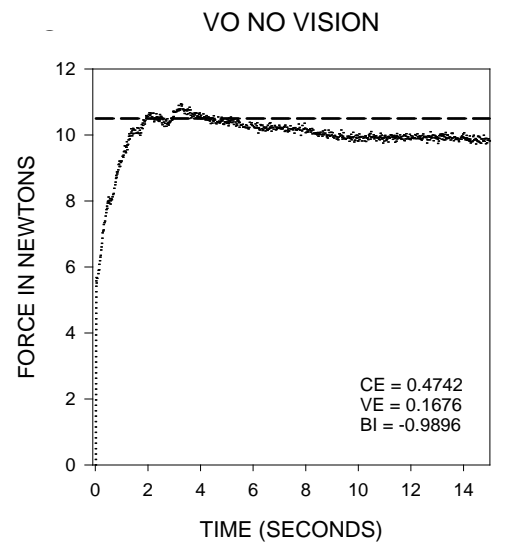

(c)

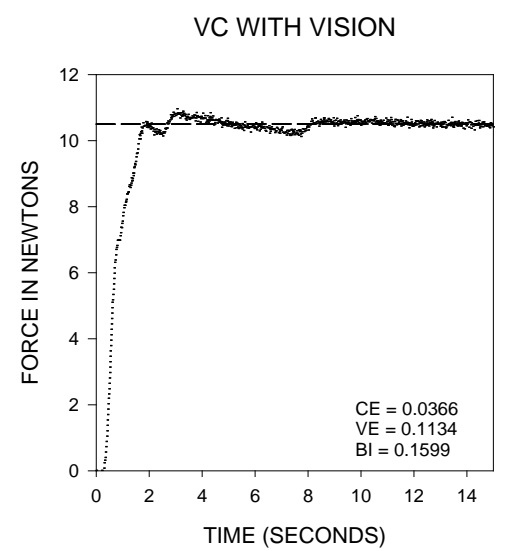

(b)

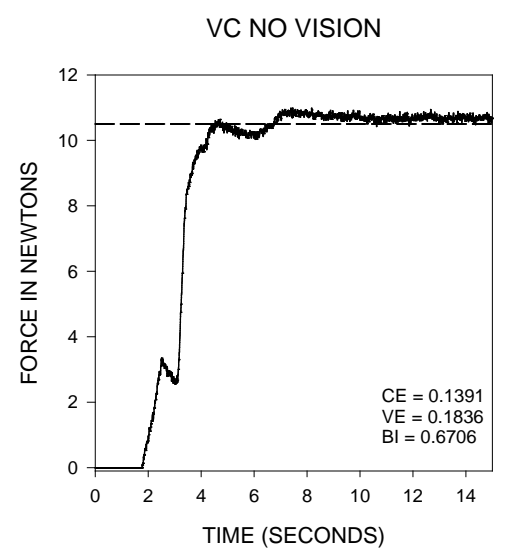

(d)

Figure 2. Examples of the force output produced by using the voluntary opening prehensor (left two panels) and the voluntary closing prehensor (right two panels). The two upper panels represent the force output for the voluntary opening and voluntary closing prehensors with vision and the lower two panels represent the force output for the voluntary opening and voluntary closing prehensors with no vision. The inset values give the corresponding $\mathrm{CE}, \mathrm{VE}$, and $\mathrm{BI}$ for each trial. 


\section{Results}

Each position $\times$ force $\times$ vision condition was repeated for three trials. No differences were observed between the $90^{\circ}$ and $180^{\circ}$ elbow positions, so results were collapsed across position for corresponding force $\times$ vision conditions.

\subsection{Constant Error}

The voluntary closing device was more accurate than the voluntary opening device. Figure 3 shows the mean $\mathrm{CE}+\mathrm{SEM}$ for the 0.49 and $10.5 \mathrm{~N}$ vision and no vision trials (Figure 3 ).

A t-test on the 6 repeated trials of each condition revealed that the CE for the voluntary closing device was lower than for the voluntary opening for both the vision $(t=-2.48, \mathrm{p}<0.05)$ and no-vision $(\mathrm{t}=-2.18, \mathrm{p}<0.05)$ conditions at the 10.5 newton target. These results indicate that the participant was more accurate when using the voluntary closing device compared to his native voluntary opening device.

\subsection{Variable Error}

The voluntary closing device was less variable than the voluntary opening device. Figure 4 shows the mean VE + SEM for the 0.49 and $10.5 \mathrm{~N}$ vision and no vision trials (Figure 4).
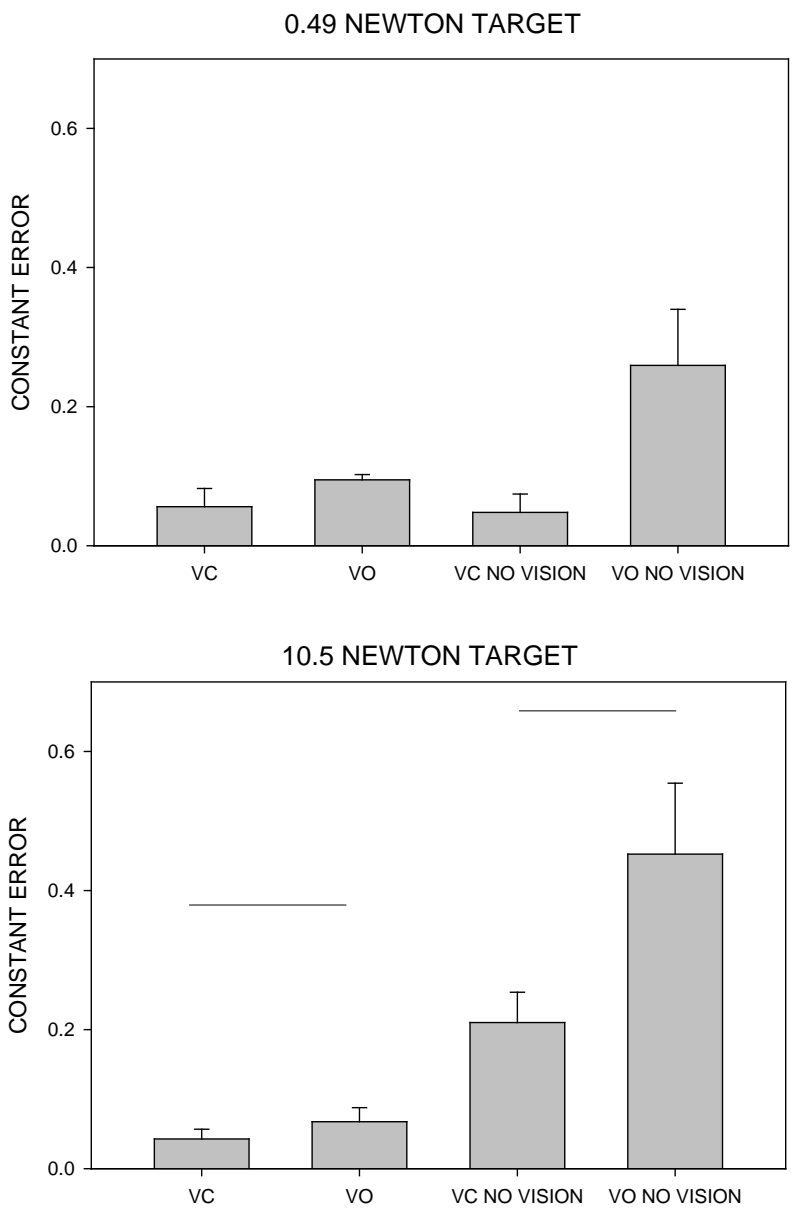

Figure 3. Mean + SEM for constant error. The voluntary opening prehensor had significantly higher constant error for the vision conditions $(\mathrm{t}=-2.48, \mathrm{p}<0.05)$ and the no-vision condition $(\mathrm{t}=-2.18, \mathrm{p}<0.05)$ at the $10.5 \mathrm{~N}$ target. Horizontal lines represent comparisons where there was a significant difference as elucidated by a t-test. 

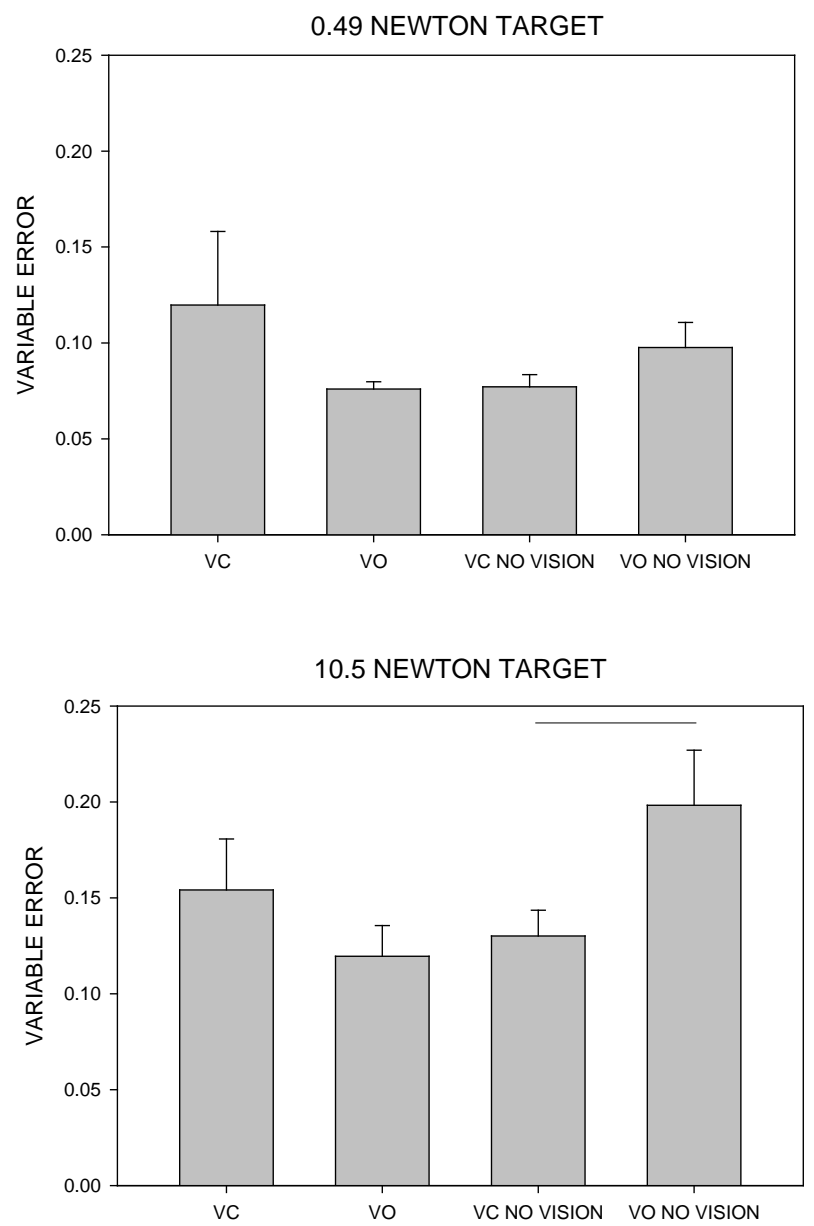

Figure 4. Mean + SEM for variable error. The voluntary opening prehensor had significantly higher variable error for the novision condition $(\mathrm{t}=-2.15, \mathrm{p}<0.05)$ at the $10.5 \mathrm{~N}$ target. Horizontal lines represent comparisons where there was a significant difference as elucidated by a t-test.

A t-test on the 6 repeated trials of each condition revealed that the voluntary closing was less variable than the voluntary opening for the condition with no-vision $(\mathrm{t}=-2.15, \mathrm{p}<0.05)$. These results indicate that the participant was less variable when using the voluntary closing device compared to his native voluntary opening device.

\subsection{Bias Index}

The voluntary closing device was less biased than the voluntary opening device. Figure 5 shows mean BI + SEM for the 0.49 and $10.5 \mathrm{~N}$ vision and no vision trials (Figure 5).

A t-test on the 6 repeated trials of each condition revealed that the voluntary closing was on target more than the voluntary opening for the no vision conditions at 0.49 newtons $(t=2.13, \mathrm{p}<0.05)$ as well as the vision condition at 10.5 newtons $(\mathrm{t}=-2.614, \mathrm{p}<0.05)$.

\section{Discussion}

The purpose of the present study was to determine how well an experienced user of the voluntary opening prosthesis could adapt to a voluntary closing prosthesis without training. The rationale for the study was based on the muscular force-grip force compatibility hypothesis [7]. The findings were consistent with the hypothesis that performance would be better with the prehensor that had the highest degree of compatibility between muscular force and grip force. When the participant used the voluntary closing prehensor, force output was more accurate, 

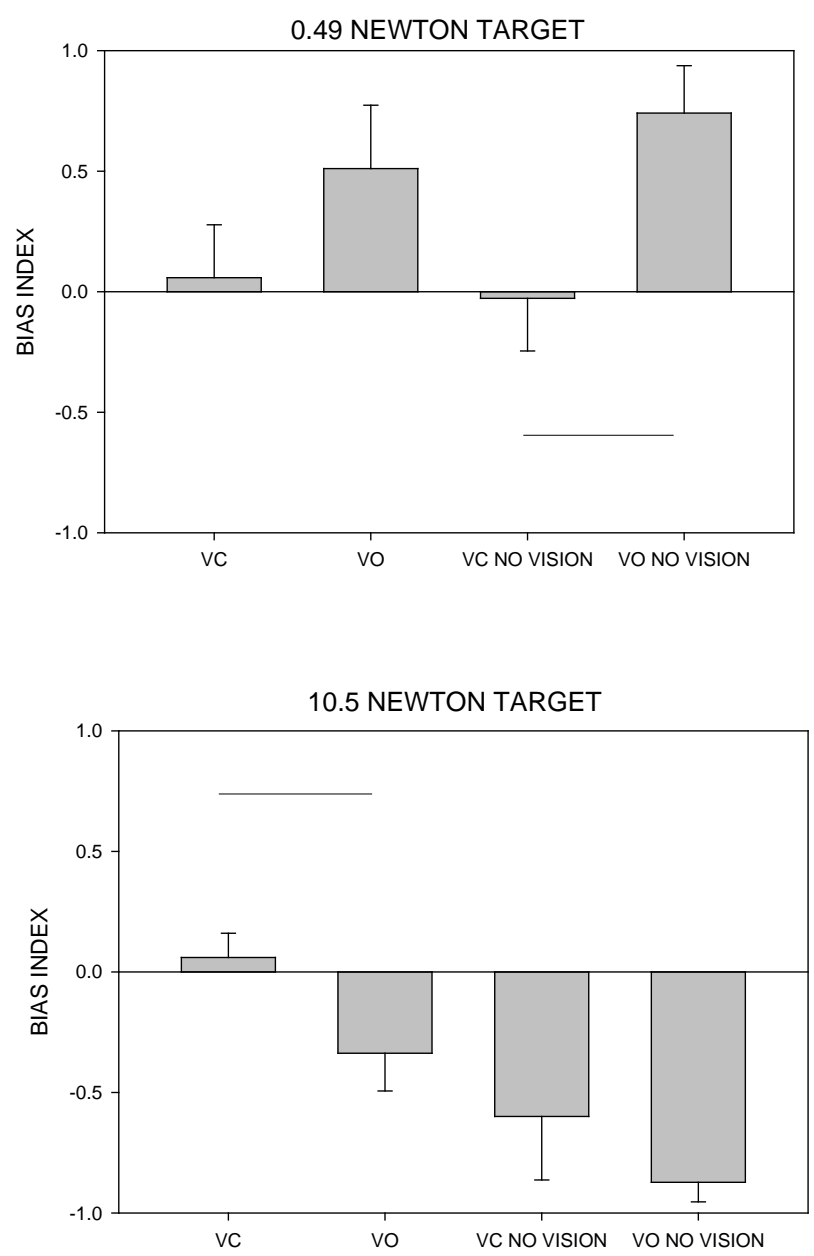

Figure 5. Mean + SEM for bias index. The voluntary opening prehensor overshot the target more than the voluntary closing prehensor for the no vision condition at the $0.49 \mathrm{~N}$ target $(\mathrm{t}=$ $2.13, \mathrm{p}<0.05$ ). The voluntary opening prehensor undershot the target more than the voluntary closing prehensor for the vision condition at the $10.5 \mathrm{~N}$ target $(\mathrm{t}=-2.614, \mathrm{p}<0.05)$. Horizontal lines represent comparisons where there was a significant difference as elucidated by a t-test.

more consistent, and less biased. The superiority of the voluntary closing prehensor was most apparent when the accuracy of force output was examined in the no-vision condition.

The muscular force-grip force compatibility hypothesis is based on the compatibility between the amount of muscular force required to produce a grip and the resulting force on the object that is being gripped. The compatibility in the anatomical hand is high because grip force increases as the muscular force needed to create the grip increases. Furthermore, increases in grip force output have been shown to correlate with the frequency of cortical oscillation suggesting that central mechanisms may exist to accommodate compatibility between force effort and force output [11]. The same type of compatibility is present in the voluntary closing prehensor. As the muscular force needed to increase cable tension increases, the resulting grip force produced by the prehensor increases. In contrast, the compatibility is low for the voluntary opening prehensor because an increase in grip force is accomplished by releasing tension on the cabling system, which is accomplished by decreasing the amount of muscular force. The current findings are intriguing because the participant performed best in the high compatibility prehensor (with the voluntary closing prehensor) even though he had over three decades of experience with the less compatible prehensor (the voluntary opening prehensor). Clearly, then, the compatibility between muscular force and force output was a more important contributor to performance than previous expe- 
rience. In other words, compatibility was more important than specificity training [8]-[10].

Despite the aforementioned conclusion, experience may play an important role in how the compatibility issue is handled by the user. We would predict that inexperienced prosthetic users would show much greater performance differences between the voluntary opening and voluntary closing prehensor. Compatibility is likely to be a much bigger issue in tasks that require unexpected changes in grip force output in response to changing task demands (e.g., maintaining the grip on an object that suddenly slips from the grasp). Thus, prosthetists and occupational therapists should be mindful that the person with a recent amputation may have greater difficulty learning to use a voluntary opening prosthesis than a voluntary closing prosthesis in tasks that require grip force modulation because of the patient's unfamiliarity with the lack of compatibility between muscular force and force output in the voluntary opening prehensor. This suggestion could be incorporated into other recommendations for prosthetic training [1] [12]. The current participant has likely overcome some of the compatibility limitations associated with his voluntary opening prehensor as a function of his extensive experience. However, it should be pointed out that in different types of incompatibility, such as stimulus-response incompatibility and the Stroop effect, it has been shown that the negative consequences of incompatibility, may be reduced but may not be entirely eliminated with extensive practice [13] [14].

In addition, it is pertinent to note that the availability of vision also likely makes a contribution to the user's ability to overcome these limitations [15]. The difference between the two prehensors was more apparent when vision was not available in the current experiment. Visual feedback enables the user to immediately correct any deviations from the intended output, whereas the absence of visual feedback requires the user to rely on potentially less salient sources of feedback from the musculoskeletal system.

This study is not without its limitations. One limitation is that case studies do not lend themselves well to generalization to the larger population. It is possible that different lifelong voluntary opening prehensor users may have different results compared to our participant. Nonetheless, the results presented in this study are intriguing given the superior performance of the voluntary closing device on each measure of accuracy and variability. Additionally, we assessed static force production for two highly-relevant forces. The performance on producing those two forces may not generalize to every grip force that an upper extremity prosthetic limb user may produce in the workplace or in activities of daily living. Further studies should be performed to assess performance for modulating grip force as well as forces that are greater than the two we tested.

\section{Conclusion}

In summary, we observed that an experienced user of voluntary opening prostheses actually performed better using a voluntary closing prosthesis in a task requiring the control of grip pressure. Whether these findings can be found with other experienced voluntary opening prostheses users and whether the muscle force-grip force incompatibility can be observed with inexperienced users of the voluntary opening prosthesis is an open question. Future studies might also focus on expanding the present findings to tasks that require more demanding grip force modulation.

\section{Acknowledgements}

We would like to thank William Ulrich for the drawings in Figure 1.

\section{References}

[1] González-Fernández, M. (2014) Development of Upper Limb Prostheses: Current Progress and Areas for Growth. Archives of Physical Medicine and Rehabilitation, 95, 1013-1014. http://dx.doi.org/10.1016/j.apmr.2013.11.021

[2] Frey, D.D., Carlson, L.E. and Ramaswamy, V. (1995) Voluntary-Opening Prehensors with Adjustable Grip Force. Journal of Prosthetics and Orthotics, 7, 124-131. http://dx.doi.org/10.1097/00008526-199507040-00004

[3] De Visser, M. and Herder, J.L. (2000) Re-Directed Design of a Voluntary Closing Hand Prosthesis. Journal of Rehabilitation Research and Development, 37, 261-271.

[4] Murphy, E.F. (1964) Manipulators and Upper-Extremity Prosthetics. Bulletin of Prosthetics Research, 107-117.

[5] Plettenburg, D.H. and Herder, J.L. (2003) Voluntary Closing: A Promising Opening in Hand Prosthetics. Technology and Disability, 15, 85-94.

[6] Radocy, B. (1986) Voluntary Closing Control: A Successful New Design Approach to an Old Concept. Clinical Pros- 
thetics and Orthotics, 10, 82-86.

[7] Wallace, S.A., Anderson, D.I., Trujillo, M. and Weeks, D.L. (2005) Upper Extremity Artificial Limb Control as an Issue Related to Movement and Mobility in Daily Living. Quest, 57, 124-137. http://dx.doi.org/10.1080/00336297.2005.10491846

[8] Proteau, L. and Marteniuk, R.G. (1993) Static Visual Information and the Learning and Control of a Manual Aiming Movement. Human Movement Science, 12, 515-536. http://dx.doi.org/10.1016/0167-9457(93)90003-8

[9] Proteau, L., Tremblay, L. and DeJaeger, D. (1998) Practice Does Not Diminish the Role of Visual Information in On-Line Control of a Precision Walking Task: Support for a Specificity of Practice Hypothesis. Journal of Motor Behavior, 30, 143-150. http://dx.doi.org/10.1080/00222899809601331

[10] Tremblay, L. and Proteau, L. (1998) Specificity of Practice: The Case of Powerlifting. Journal of Motor Behavior, Research Quarterly for Exercise and Sport, 69, 284-289. http://dx.doi.org/10.1080/02701367.1998.10607695

[11] Rong, Y., Han, X., Hao, D., Cao, L., Wang, Q., Li, M., Duan, L. and Zeng, Y. (2014) Applying Support Vector Regression Analysis on Grip Force Level-Related Corticomuscular Coherence. Journal of Computational Neuroscience, $1-11$.

[12] Atkins, D. (2002) Adult Upper Limb Prosthetic Training. In: Bowker, H.K. and Michael, J.W., Eds., Adult Upper Limb Prosthetic Training, Rosement.

[13] Davidson, D.J., Zacks, R.T. and Williams, C.C. (2003) Stroop Interference, Practice and Aging. Aging, Neuropsychology, and Cognition: A Journal on Normal and Dysfunctional Development, 10, 85-98.

[14] Dutta, A. and Proctor, R.W. (1992) Persistence of Stimulus-Response Compatibility Effects with Extended Practice. Journal of Experimental Psychology: Learning, Memory and Cognition, 18, 801-809. http://dx.doi.org/10.1037/0278-7393.18.4.801

[15] Engeberg, E.D. and Meek, S. (2012) Enhanced Visual Feedback for Slip Prevention with a Prosthetic Hand. Prosthetics and Orthotics International, 36, 423-429. http://dx.doi.org/10.1177/0309364612440077 
Scientific Research Publishing (SCIRP) is one of the largest Open Access journal publishers. It is currently publishing more than 200 open access, online, peer-reviewed journals covering a wide range of academic disciplines. SCIRP serves the worldwide academic communities and contributes to the progress and application of science with its publication.

Other selected journals from SCIRP are listed as below. Submit your manuscript to us via either submit@scirp.org or Online Submission Portal.
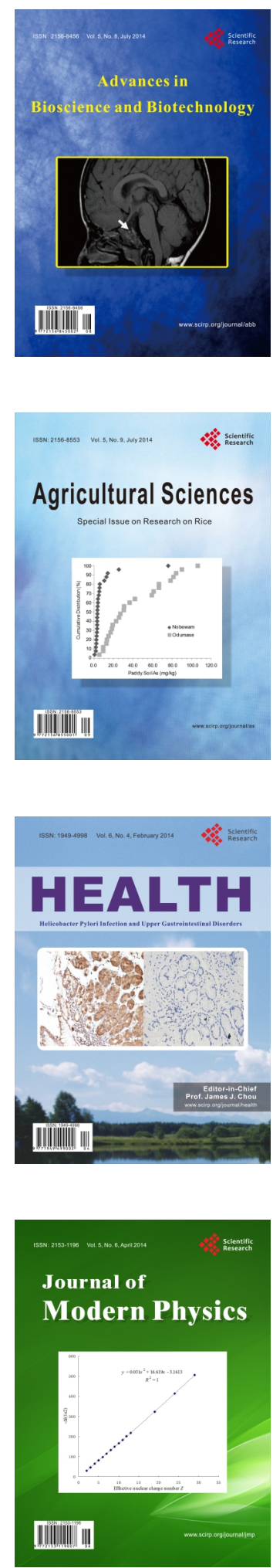
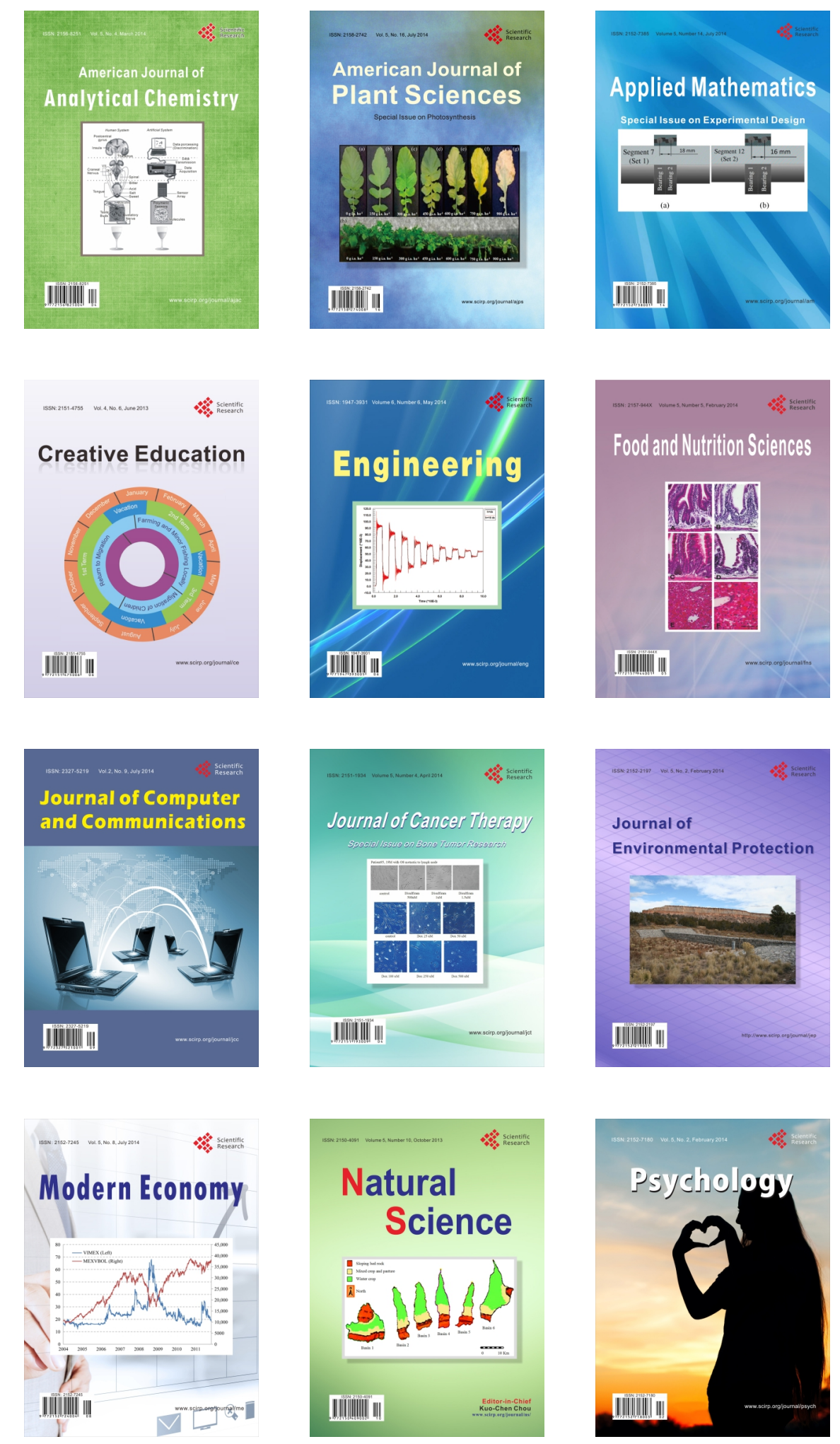